\title{
A Comparative Study of Treatment-Emergent Adverse Events Following Use of Common Bowel Preparations Among a Colonoscopy Screening Population: Results from a Post-Marketing Observational Study
}

\author{
Kathryn Anastassopoulos ${ }^{2} \cdot$ Francis A. Farraye ${ }^{3} \cdot$ Tyler Knight $^{2} \cdot$ Sam Colman $^{2}$ • \\ Mark vB. Cleveland $^{1} \cdot$ Russell W. Pelham ${ }^{1}$
}

Received: 3 March 2016/Accepted: 26 May 2016/Published online: 9 June 2016

(c) The Author(s) 2016. This article is published with open access at Springerlink.com

\begin{abstract}
Background Colonoscopy may be one of the most frequent elective procedures in older adults and is associated with a low occurrence of complications. However, reduction of risks attributable to the bowel preparation may be achieved with the use of effective and safer products.

Aim The aim of this study was to examine the incidence of treatment-emergent adverse events (TEAEs) associated with SUPREP ${ }^{\circledR}$ [oral sulfate solution (OSS)] and other common prescription bowel preparations (non-OSS).

Methods This real-world, observational study used deidentified health insurance claims and laboratory results to identify TEAEs in the 3 months following screening colonoscopy in adults with a prescription for a bowel preparation in the prior 60 days. The unadjusted and adjusted (controlling for patient risk factors) cumulative incidences of TEAEs were estimated using Kaplan-Meier and Poisson regression, respectively.

Results Among patients $\geq 45$ years, the overall cumulative incidence was significantly lower $(p<0.001)$ in the OSS cohort than in the non-OSS cohort (unadjusted: $2.31 \mathrm{vs.}$ $2.89 \%$; adjusted: 1.61 vs. $1.95 \%$ ), with significantly lower acute cardiac conditions ( 1.56 vs. $1.90 \% ; p<0.001)$, renal
\end{abstract}

Prior Presentation of Data: American College of Gastroenterology Annual Scientific Meeting (2013).

Russell W. Pelham

RPelham@braintreelabs.com

Braintree Laboratories Inc., Braintree, MA, USA

2 Health Economics and Outcomes Research, Covance Market Access Services Inc., Gaithersburg, MD, USA

3 Section of Gastroenterology, Boston Medical Center, Boston University School of Medicine, Boston, MA, USA failure/other serious renal diseases (OSS: $0.21 \%$, non-OSS: $0.32 \% ; p<0.001$ ), and serum electrolyte abnormalities (OSS: $0.39 \%$, non-OSS: $0.49 \% ; p=0.017$ ). There were no significant differences between cohorts in death, seizure disorders, aggravation of gout, and ischemic colitis. Results were similar in the adjusted cumulative incidences.

Conclusions In actual use, the overall cumulative incidence of TEAEs was significantly lower in the OSS cohort, demonstrating that OSS is as safe as, or possibly safer than, non-OSS prescription bowel preparations.

Keywords Colonoscopy · Bowel preparation · Adverse event

\section{Introduction}

Colonoscopy may be one of the most frequent elective procedures, particularly in adults aged 45 years and older in which it is part of a recommended colorectal cancer screening program [1]. Complications from colonoscopy and the bowel preparation that precedes the procedure occur at a low frequency [2]; however, reduction of risks that are attributable to the bowel preparation may be achieved with the use of effective and safer products [3].

To date, the safest bowel preparations are generally recognized to be the isotonic formulations composed of polyethylene glycol (PEG) and electrolytes, which have been in use since the early 1980s. These preparations induce a voluminous osmotic diarrhea and are designed to prevent electrolyte and water gains or losses [4, 5]. However, adequate cleansing for colonoscopy with these preparations requires ingestion of a large volume of solution, typically about $4 \mathrm{~L}$. In response to patient demands for lower volume and more tolerable preparations, a small 
volume hypertonic saline preparation based on sodium phosphate was promoted in the 1990s [6]. This product similarly induced an osmotic diarrhea, but unlike the isosmotic preparations, supplemental water was required to prevent dehydration. Rare case reports of acute renal failure eventually emerged followed by publication of kidney biopsy data showing renal calcification in association with use of the phosphate preparations [7]. The United States (US) Food and Drug Administration (FDA) subsequently issued a warning resulting in the voluntary removal from the market of the liquid form of the sodium phosphate bowel preparation and a "black box" warning for the tablet preparations [8].

A new hypertonic saline preparation has been introduced based on a combination of the sulfate salts of sodium, potassium, and magnesium (SUPREP ${ }^{\circledR}$ [oral sulfate solution (OSS)], Braintree Laboratories Inc.) [9]. Studies of the physiology of both phosphate and sulfate hypertonic preparations in normal volunteers showed that the use of the phosphate preparation, but not the sulfate formulation, markedly increased urine phosphate concentration with a corresponding increase in propensity for formation of calcium precipitates, despite vigorous hydration of study subjects [10]. Clinical studies of the sulfate formulation have demonstrated its safety and efficacy under the rigorous conditions inherent in formal clinical trials where it appears to be equally safe to the PEG-based preparations [9-11]. However, real-world clinical experience can vary in ways not observed within the confines of clinical trials. For example, based on the phosphate experience, because the product requires ingestion of supplemental water to replace fluid loss induced by the osmotic diarrhea, some patients may not consume sufficient replacement water, which results in problems associated with dehydration. Additionally, rare unanticipated outcomes are more likely to be detected in large, observational studies.

This study compared the incidence of treatment-emergent adverse events (TEAEs) which may occur in association with the recently approved OSS bowel preparation to other common bowel preparations in patients aged 45 years or older who underwent screening colonoscopy. This is the first report that examines the incidence of TEAEs in clinical practice for any bowel preparation to other available prescription bowel preparations in a large population of screening patients.

\section{Methods}

\section{Study Design}

This retrospective, real-world, observational study was undertaken as a post-marketing pharmacovigilance safety study of OSS in screening colonoscopy patients. The study was approved by the New England Institutional Review Board and was agreed by the FDA. Data were obtained from a de-identified health insurance claims data source (MarketScan ${ }^{\circledR}$ Commercial Claims and Encounters Database and Medicare Supplemental and Coordination of Benefits Database, Truven Health Analytics, Inc.). Laboratory results data were also obtained (Truven Health Analytics, Inc.). Health insurance claims included inpatient, outpatient, and prescription drug claims for over 170 million individuals in the USA annually from approximately 90 large employers and health plans, with insurance provided under various fee-for-service and capitated health plans. The data include commercially insured beneficiaries aged 0-64 years and beneficiaries aged 65 and older with Medicare coverage in addition to commercial insurance. The study period included claims from August 1, 2009, through December 31, 2011, with an observation period from August 1, 2010, through December 31, 2011.

The primary objective was to describe and compare, in real-world settings, the incidence of TEAEs observed associated with OSS to other prescription bowel preparations occurring at any time in the 3 months following administration for screening colonoscopy in an adult population in the USA.

\section{Study Population}

Patients who received a bowel preparation followed by a screening colonoscopy were included in the study. Specifically, patients who met the following criteria were included:

- had a claim for a screening colonoscopy;

- had a claim for one of the following bowel preparations within the 60 days prior to the screening colonoscopy: OSS (i.e., SUPREP ${ }^{\circledR}$ ); PEG-3350 and electrolytes for oral solution (generic or Clenz-Lyte ${ }^{\mathrm{TM}}$ [Paddock Laboratories Inc.], Colyte ${ }^{\circledR}$ [Meda Pharmaceuticals Inc.], GaviLyte-C, GaviLyte-G, GaviLyte-N, GoLytely ${ }^{\circledR}$ [Braintree Laboratories Inc.], HalfLytely ${ }^{\circledR}$ [Braintree Laboratories Inc.], Moviprep ${ }^{\circledR}$ [Salix Pharmaceuticals Inc.], NuLytely ${ }^{\circledR}$ [Braintree Laboratories Inc.], Trilyte ${ }^{\circledR}$ [Wallace Pharmaceuticals]); sodium phosphate monobasic monohydrate, USP, and sodium phosphate dibasic anhydrous, USP (Osmoprep ${ }^{\circledR}$, Salix Pharmaceuticals Inc.), or sodium phosphate monobasic monohydrate, sodium phosphate dibasic anhydrous (Visicol $^{\circledR}$, Salix Pharmaceuticals Inc.);

- had been continuously enrolled in their insurance plan during the 12 months prior to and 3 months following and including the administration of the bowel preparation; and 
- were 18 years of age or older on the day of the administration of the bowel preparation.

Patients were excluded from the study if they had a claim that included:

- a prescription for both OSS and one of the other bowel preparations within the 60 days prior to screening colonoscopy (excluded from primary analysis); or

- evidence that the colonoscopy was not a screening colonoscopy such as:

- a colon polypectomy in the 12 months prior to the administration of the bowel preparation; or

- a diagnosis of a gastrointestinal cancer (including colon, esophagus, rectum, small intestine, and stomach) during the 12 months prior to the administration of the bowel preparation.

Screening colonoscopy procedures were identified by the following Healthcare Common Procedure Coding System (HCPCS) codes or combined Current Procedural Terminology (CPT) and International Classification of Diseases, 9th Revision, Clinical Modification (ICD-9-CM) diagnosis codes [12]:

- HCPCS: G0105 (colorectal cancer screening; colonoscopy on individual at high risk).

- HCPCS: G0121 (colorectal cancer screening; colonoscopy on individual not meeting criteria for high risk).

- CPT: 45378 (colonoscopy, flexible, proximal to splenic flexure; with or without colon decompression), 45380 (colonoscopy, flexible, proximal to splenic flexure; with biopsy), 45384 (colonoscopy, flexible, proximal to splenic flexure; with removal of tumor, polyp, or other lesion by hot biopsy forceps or bipolar cautery), 45385 (colonoscopy, flexible, proximal to splenic flexure; with removal of tumor, polyp, or other lesions by snare technique), or 45392 (colonoscopy, flexible, proximal to splenic flexure; with transendoscopic ultrasoundguided intramural or transmural fine needle aspiration/ biopsy) and ICD-9-CM diagnosis code: V76.51 (special screening for malignant neoplasm-colon) or V16.0 (family history of malignant neoplasm-gastrointestinal tract).

The bowel preparations were identified by National Drug Code.

Patients meeting the inclusion and exclusion criteria who had a prescription claim for OSS were included in the OSS cohort. Patients with a prescription claim for one of the other bowel preparations were included in the non-OSS cohort.

\section{Identification of TEAEs}

A TEAE was defined as an event that occurred any time in the 3 months following administration of the bowel preparation for a screening colonoscopy (i.e., follow-up period) and did not occur in the 12 months prior to administration (i.e., baseline period). The 3-month period following administration was chosen as an appropriate follow-up period to observe the TEAEs of interest, particularly renal failure. This period is two months longer than the observation period in a study of safety of bowel preparations in the Medicare population [12]. A 3-month period was expected to be adequate, as most cases $(85.7 \%)$ of acute renal failure occur during the first 2 months after oral phosphate solution [7]. TEAEs that were examined have been historically associated with bowel preparations and were identified by primary and secondary ICD-9-CM diagnosis codes or out-of-range laboratory values [13]. TEAEs identified by diagnosis codes included ischemic colitis [14], aggravation of gout, acute cardiac conditions or events, renal failure and other serious renal diseases, seizure disorders, and serum electrolyte abnormalities. TEAEs identified by laboratory values included troponin leak, elevated creatinine kinase, serum electrolyte abnormalities, and serum uric acid. The codes used to identify the TEAEs are provided in Exhibit 1 of Appendix. Death was identified within the inpatient setting using an indicator for death on the discharge status of the inpatient claim.

Patient risk factors associated with the TEAEs were also identified by ICD-9-CM diagnosis codes during the baseline period. These included arteriosclerosis, brain cancer, diabetes mellitus, gout, heart failure, hyperlipidemia, hypertension, hypotension, liver disease, obesity, peripheral artery disease, previous abdominal surgery (colectomy, colostomy, gastric bypass, stomach stapling), renal disease, stroke, thiazide diuretics use, thyroid disease, and vasculitis [15].

\section{Analytic Methods}

Descriptive statistics (i.e., means, standard deviations, medians, ranges) on the demographic and clinical risk factors observed prior to the administration of the bowel preparation were calculated for each bowel preparation cohort (i.e., OSS and non-OSS). To examine the incidence of each TEAE in real-world settings, the unadjusted cumulative incidence of each TEAE and a $95 \%$ confidence interval (CI) were calculated using the Kaplan-Meier method. Differences between the two cohorts were tested using the log-rank Chi-square. To account for any 
differences between the two cohorts in demographic or clinical risk factors prior to the administration of the bowel preparation, an adjusted cumulative incidence was calculated for each TEAE. Adjusted cumulative incidences and $95 \%$ CIs were calculated using Poisson regression, controlling for demographic and clinical risk factors potentially associated with the bowel preparation and each TEAE, as applicable. The clinical risk factors included in the Poisson regression for each TEAE are provided in Exhibit 1 of Appendix. Demographic and clinical risk factors were removed from the Poisson model if its parameter estimate was not significant (i.e., $p$ value greater than 0.10). Follow-up time was used as on offset in the Poisson regression. Differences in the adjusted incidences between the two cohorts were tested using the Wald Chisquare from the Poisson regression. When no events were observed within a cohort, the Clopper-Pearson method was used to calculate an exact $95 \%$ CI. The Fisher's exact test was used to calculate a $p$ value when no events were observed in either cohort. Patients with less than 3 months of follow-up due to death, but had no other TEAEs, were censored for the calculation of incidence of each TEAE other than death. The Benjamini-Hochberg false discovery rate procedure $[16,17]$ was used to control for multiple comparisons for the unadjusted and adjusted incidences in the main analysis, maintaining a type I error rate of 0.05 which resulted in a claim of significance if the $p$ value was less than 0.018 among the unadjusted incidences and less than 0.014 among the adjusted incidences.

The main analysis focused on patients aged 45 years or older because screening colonoscopy is indicated for this age group [1]. A secondary analysis examined patients aged 18-44 years. Analyses were performed using SAS Software, version 9.1 or higher (SAS Institute, Cary, North Carolina).

\section{Results}

A total of 835,219 patients were identified as having a screening colonoscopy during the study period. Figure 1 presents the effect of the inclusion and exclusion criteria on the study population. Approximately $25 \%$ of patients were excluded because they were not continuously enrolled during the 12 months prior to and 3 months following and including the administration of the bowel preparation so their TEAEs could not be assessed. Another $39 \%$ of patients were excluded because they had no pharmacy claim for a prescription bowel preparation, perhaps due to the physician providing bowel preparation samples and/or recommending over-the-counter products. Thus, just over one-third $(35.8 \%)$ of these patients met the inclusion and exclusion criteria for this study, with 287,323 (96.0\%) of these patients aged 45 years or older (OSS: 31,885; nonOSS: 255,438).

Demographic and clinical characteristics of these patients are presented in Table 1. The average age of patients was slightly lower in the OSS cohort compared to the non-OSS cohort (56.6 vs. 57.5 years; $p<0.001$ ) (Table 2). The percent of males was similar between the two cohorts (OSS: $47.1 \%$, non-OSS: $46.7 \% ; p=0.178$ ). The most common $(\geq 5 \%)$ TEAE risk factors were arteriosclerosis, diabetes, hyperlipidemia, hypertension, thiazide diuretics use, and thyroid disease. For each TEAE risk factor, except brain cancer, previous abdominal surgery, thyroid disease, and vasculitis, the percent of patients differed significantly ( $p<0.05$ ) between cohorts; however, the difference was approximately $2 \%$ or less for all TEAE risk factors.

\section{Main Analysis}

\section{Unadjusted Incidence}

The unadjusted cumulative incidences of the TEAE categories identified by diagnosis codes are presented in Table 2, and the incidences of the individual TEAEs that made up the categories are presented in Exhibit 2 of Appendix. The overall cumulative incidence was significantly lower in the OSS cohort (2.31 vs. $2.89 \%$; $p<0.001)$.

Among the TEAE categories, the incidence of acute cardiac conditions was also significantly lower in the OSS cohort $(1.56$ vs. $1.90 \% ; p<0.001)$ with the majority of patients with acute cardiac events experiencing arrhythmia (OSS: 230 [46.1\%], non-OSS: 2250 [46.4 \%]; Appendix, Exhibit 2) followed by abnormal electrocardiogram (OSS: 141 [28.3 \%], non-OSS: 1465 [30.2\%]; Appendix, Exhibit 2). Additionally, renal failure/other serious renal diseases (OSS: $0.21 \%$, non-OSS: $0.32 \% ; p<0.001$ ) and serum electrolyte abnormalities (OSS: $0.39 \%$, non-OSS: $0.49 \%$; $p=0.017)$ were significantly lower in the OSS cohort.

The cumulative incidences were not significantly different for the following other TEAE categories: seizure disorders (OSS: $0.27 \%$, non-OSS: $0.34 \% ; p=0.040$ ), aggravation of gout (OSS: $0.24 \%$, non-OSS: $0.28 \%$; $p=0.151$ ), death (OSS: $0.01 \%$, non-OSS: $0.02 \%$; $p=0.366$ ), and ischemic colitis (OSS: $0.02 \%$, non-OSS: $0.02 \% ; p=0.722$ ).

Among the patients with laboratory data (OSS: 653 and non-OSS: 14,432), the incidences were also not significantly different for the TEAE categories of serum electrolyte abnormalities/serum uric acid (OSS: $0.92 \%$, nonOSS: $1.52 \% ; p=0.218)$, elevated creatine kinase (OSS: $0.00 \%$, non-OSS: $0.03 \% ; p=1.000)$, and troponin leak (OSS: $0.00 \%$, non-OSS: $0.00 \% ; p=1.000$ ). 


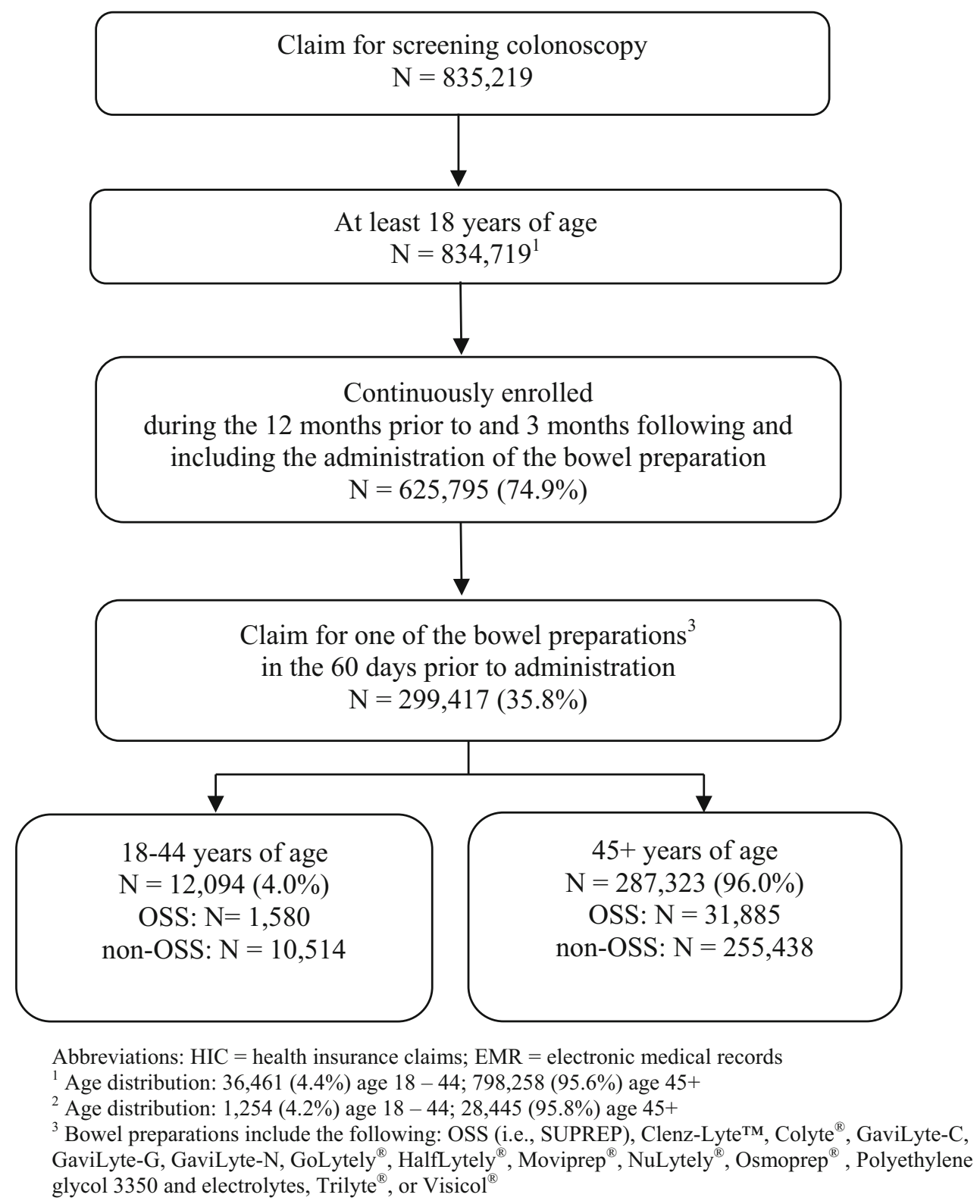

Fig. 1 Study population

\section{Adjusted Incidence}

The adjusted cumulative incidences of the TEAEs identified by diagnosis codes are presented in Table 3, and the incidences of the individual TEAEs that made up the categories are presented in Exhibit 3 of Appendix. The overall cumulative incidence was significantly lower in the OSS cohort (1.61 vs. $1.95 \% ; p<0.001)$. Among the TEAE categories, the incidence of acute cardiac conditions (1.23 vs. $1.47 \% ; p<0.001)$ and renal failure/other serious renal diseases (OSS: $0.10 \%$, non-OSS: $0.15 \% ; p=0.004$ ) was also significantly lower in the OSS cohort. The cumulative incidences were not significantly different, for the following other TEAE categories: seizure disorders (OSS: $0.26 \%$, non-OSS: $0.32 \% ; p=0.046$ ), serum electrolyte abnormalities (OSS: $0.34 \%$, non-OSS: $0.41 \%$; $p=0.048$ ), aggravation of gout (OSS: $0.12 \%$, non-OSS: $0.14 \% ; p=0.233$ ), death (OSS: $0.01 \%$, non-OSS: $0.02 \% ; p=0.369$ ), and ischemic colitis (OSS: $0.02 \%$, non-OSS: $0.02 \% ; p=0.743$ ).

Among the patients with laboratory data, the incidences were not significantly different for the TEAE categories of serum electrolyte abnormalities/serum uric acid (OSS: $0.86 \%$, non-OSS: $1.37 \% ; p=0.257)$, elevated creatine kinase (OSS: $0.00 \%$, non-OSS: $0.03 \% ; p=1.000$ ), and troponin leak (OSS: $0.00 \%$, non-OSS: $0.00 \% ; p=1.000$ ). 
Table 1 Demographic and baseline clinical risk factors of an adult screening colonoscopy population

\begin{tabular}{lcc}
\hline Characteristic & OSS $(N=31,885)$ & non-OSS $(N=255,438)$ \\
\hline Age, mean (sd) & $56.6(6.4)$ & $57.5(7.3)$ \\
Male, $n(\%)$ & $15,026(47.1)$ & $119,357(46.7)$ \\
TEAE risk factors & & \\
Arteriosclerosis, $n(\%)$ & $1657(5.2)$ & $15,704(6.1)$ \\
Brain cancer, $n(\%)$ & $12(0.0)$ & $114(0.0)$ \\
Diabetes, $n(\%)$ & $3982(12.5)$ & $35,687(14.0)$ \\
Heart failure, $n(\%)$ & $237(0.7)$ & $2646(1.0)$ \\
Hyperlipidemia, $n(\%)$ & $12,397(38.9)$ & $100,904(39.5)$ \\
Hypertension, $n(\%)$ & $10,836(34.0)$ & $92,760(36.3)$ \\
Hypotension, $n(\%)$ & $125(0.4)$ & $1357(0.5)$ \\
Liver disease, $n(\%)$ & $871(2.7)$ & $7503(2.9)$ \\
Obesity, $n(\%)$ & $838(2.6)$ & $8285(3.2)$ \\
Peripheral artery disease, $n(\%)$ & $247(0.8)$ & $2414(0.9)$ \\
Previous abdominal surgery, $n(\%)$ & $970(3.0)$ & $7735(3.0)$ \\
Stroke, $n(\%)$ & $811(2.5)$ & $7545(3.0)$ \\
Thiazide diuretics use, $n(\%)$ & $1808(5.7)$ & $17,424(6.8)$ \\
Thyroid disease, $n(\%)$ & $4013(12.6)$ & $31,862(12.5)$ \\
Vasculitis, $n(\%)$ & $52(0.2)$ & $378(0.1)$ \\
\hline
\end{tabular}

TEAE treatment-emergent adverse event

Table 2 Unadjusted incidence of treatment-emergent adverse events associated with bowel preparations in an adult screening colonoscopy population

\begin{tabular}{|c|c|c|c|c|c|c|c|}
\hline \multirow[t]{2}{*}{ Treatment-emergent adverse event } & \multicolumn{3}{|c|}{ OSS $(N=31,885)$} & \multicolumn{3}{|c|}{ Non-OSS $(N=255,438)$} & \multirow[t]{2}{*}{$p$ value } \\
\hline & $n$ & Incidence $(\%)$ & $95 \% \mathrm{CI}$ & $n$ & Incidence $(\%)$ & $95 \% \mathrm{CI}$ & \\
\hline \multicolumn{8}{|l|}{ Identified by diagnosis codes } \\
\hline Overall & 736 & 2.31 & $2.15,2.48$ & 7374 & 2.89 & $2.82,2.95$ & $<0.001$ \\
\hline Acute cardiac conditions or events & 499 & 1.56 & $1.43,1.71$ & 4845 & 1.90 & $1.84,1.95$ & $<0.001$ \\
\hline Aggravation of gout & 75 & 0.24 & $0.19,0.29$ & 715 & 0.28 & $0.26,0.30$ & 0.151 \\
\hline Death & 3 & 0.01 & $0.00,0.03$ & 41 & 0.02 & $0.01,0.02$ & 0.366 \\
\hline Ischemic colitis & 6 & 0.02 & $0.01,0.04$ & 56 & 0.02 & $0.02,0.03$ & 0.722 \\
\hline Renal failure and other serious renal diseases & 67 & 0.21 & $0.17,0.27$ & 817 & 0.32 & $0.30,0.34$ & $<0.001$ \\
\hline Seizure disorders & 85 & 0.27 & $0.22,0.33$ & 859 & 0.34 & $0.31,0.36$ & 0.040 \\
\hline Serum electrolyte abnormalities & 125 & 0.39 & $0.33,0.47$ & 1253 & 0.49 & $0.46,0.52$ & 0.017 \\
\hline Identified by laboratory values & \multicolumn{2}{|c|}{$N=653$} & \multicolumn{3}{|c|}{$N=14,432$} & & \\
\hline Elevated creatine kinase & 0 & 0.00 & $0.00,0.56$ & 5 & 0.03 & $0.01,0.08$ & 1.000 \\
\hline Serum electrolyte abnormalities and serum uric acid & 6 & 0.92 & $0.41,2.03$ & 219 & 1.52 & $1.33,1.73$ & 0.218 \\
\hline Troponin leak & 0 & 0.00 & $0.00,0.56$ & 0 & 0.00 & $0.00,0.03$ & 1.000 \\
\hline
\end{tabular}

The unadjusted cumulative incidence of each TEAE was calculated using the Kaplan-Meier method. Differences between the two cohorts were tested using the log-rank Chi-square test. When there were no events observed, the Clopper-Pearson method was used to calculate exact CIs. The Fisher's exact test was used to calculate the $p$ value for troponin leak

CI confidence interval

\section{Secondary Analyses}

Among patients aged $18-44$ years (OSS: 1580, non-OSS: 10,514), the overall cumulative incidences of TEAEs, identified by diagnosis codes, were similar between the two cohorts for both the unadjusted estimate (OSS: $1.33 \%$ [95 \% CI 0.87, 2.03], non-OSS: 1.46 [95 \% CI 1.25, 1.71]; $p=0.675$ ) and the adjusted estimate (OSS: $1.20[95 \% \mathrm{CI}$ $0.78,1.85]$, non-OSS: 1.30 [95 \% CI 1.09, 1.55]; $p=0.721$ ). Among patients with laboratory data (OSS: 26 
Table 3 Adjusted incidence of treatment-emergent adverse events associated with bowel preparations in an adult screening colonoscopy population

\begin{tabular}{|c|c|c|c|c|c|c|c|}
\hline \multirow[t]{2}{*}{ Treatment-emergent adverse event } & \multicolumn{3}{|c|}{ OSS $(N=31,885)$} & \multicolumn{3}{|c|}{ Non-OSS $(N=255,438)$} & \multirow[t]{2}{*}{$p$ value } \\
\hline & $n$ & Incidence $(\%)$ & $95 \% \mathrm{CI}$ & $n$ & Incidence $(\%)$ & $95 \% \mathrm{CI}$ & \\
\hline \multicolumn{8}{|l|}{ Identified by diagnosis codes } \\
\hline Overall & 736 & 1.61 & $1.49,1.74$ & 7374 & 1.95 & $1.88,2.02$ & $<0.001$ \\
\hline Acute cardiac conditions or events & 499 & 1.23 & $1.12,1.35$ & 4845 & 1.47 & $1.41,1.54$ & $<0.001$ \\
\hline Aggravation of gout & 75 & 0.12 & $0.09,0.15$ & 715 & 0.14 & $0.12,0.16$ & 0.233 \\
\hline Death & 3 & 0.01 & $0.00,0.03$ & 41 & 0.02 & $0.01,0.02$ & 0.369 \\
\hline Ischemic colitis & 6 & 0.02 & $0.01,0.04$ & 56 & 0.02 & $0.02,0.03$ & 0.743 \\
\hline Renal failure and other serious renal diseases & 67 & 0.10 & $0.08,0.13$ & 817 & 0.15 & $0.13,0.17$ & 0.004 \\
\hline Seizure disorders & 85 & 0.26 & $0.21,0.32$ & 859 & 0.32 & $0.30,0.35$ & 0.046 \\
\hline Serum electrolyte abnormalities & 125 & 0.34 & $0.28,0.40$ & 1253 & 0.41 & $0.38,0.43$ & 0.048 \\
\hline Identified by laboratory values & \multicolumn{3}{|c|}{$N=653$} & \multicolumn{3}{|c|}{$N=14,432$} & \\
\hline Elevated creatine kinase & 0 & 0.00 & $0.00,0.56$ & 5 & 0.03 & $0.01,0.08$ & 1.000 \\
\hline Serum electrolyte abnormalities and serum uric acid & 6 & 0.86 & $0.38,1.91$ & 219 & 1.37 & $1.19,1.58$ & 0.257 \\
\hline Troponin leak & 0 & 0.00 & $0.00,0.56$ & 0 & 0.00 & $0.00,0.03$ & 1.000 \\
\hline
\end{tabular}

Adjusted incidence was calculated using Poisson regression, controlling for demographic and clinical risk factors. A factor was removed from the Poisson model if its parameter estimate was not significant (i.e., $p$ value greater than 0.10). Differences between the two cohorts were tested using the Wald Chi-square. When there were no events observed, the Clopper-Pearson method was used to calculate exact CIs. The Fisher's exact test was used to calculate the $p$ value for troponin leak

CI confidence interval

and non-OSS: 582), no TEAEs were observed in the OSS cohort and nine TEAEs were observed in the non-OSS cohort for serum electrolyte abnormalities/serum uric acid.

Relative to patients aged 45 years or older, patients aged 18 to 44 years had lower overall unadjusted cumulative incidence of TEAEs identified by diagnosis codes in the OSS cohort (age 18-44: 1.33 [95\% CI 0.87, 2.03]; age $45+: 2.31$ [95\% CI 2.15, 2.48]) and in the non-OSS cohort (age 18-44: 1.46 [95\% CI 1.25, 1.71], age $45+$ : $2.89[2.82,2.95])$.

\section{Discussion}

To our knowledge, this is the largest study of post-colonoscopy TEAEs and the first to compare incidences of these TEAEs between any prescription bowel preparation product and its peers, using real-world data. This study used ICD-9 diagnosis and procedure codes appearing on health insurance claims to define just over half of the TEAEs. ICD-9 codes are recorded for billing purposes and were not developed for research studies. However, it has been shown that ICD-9 codes recorded in administrative claims have extremely high specificity in both ambulatory and inpatient settings [18-20]. The other TEAEs were defined by laboratory tests.

There is a growing need to measure results from realworld clinical practice. Randomized clinical trials typically measure short-term efficacy and safety of a product in a fairly homogenous population and under ideal, controlled conditions compared to placebo. In contrast, real clinical practice requires patient care of a heterogeneous population which is much less controlled and thus more complex. Treatment decisions made in the real clinical practice must consider a wider array of comorbid conditions, competing medications, physician preference, and possible adverse events that may be observed in a larger population or over a longer period of time than included in clinical trials. Outcomes observed in real-world settings reflect this complexity and complement the results observed in clinical trials.

In this study, the non-OSS products were primarily PEG-based preparations (generally considered to be the safest available), which constituted $94.9 \%$ of the non-OSS cohort. Although the incidences for all TEAEs were low for both cohorts, OSS demonstrated statistically significantly lower incidences compared to the non-OSS cohort for overall TEAEs and major events such as acute cardiac and serious renal events.

Unlike other bowel preparations, the safety and pharmacokinetics of OSS have been well studied in patients with renal and hepatic disease [21]. Unlike sodium phosphate preparations, OSS does not lead to formation of urinary calcium precipitates. Likewise, OSS does not alter the cardiac QTc interval, which is lengthened by oral sodium phosphate [22]. 
Similar to the FDA-approved 4-L PEG preparations, OSS had only small effects on the balance of stool fluids and electrolytes [10]. This has not been demonstrated for other marketed bowel preparations. TEAEs associated with dehydration such as syncope, hypotension, hyperosmolarity, or electrolyte imbalance were also less frequent or equivalent relative to the non-OSS cohort. The differences between OSS and the non-OSS cohorts were not due to differences in age or observed preexisting medical conditions that were accounted for in the adjusted model.

These results are somewhat surprising as the hypertonic preparation might be expected to be less safe than the nonOSS preparations (due to the risk of dehydration if patients do not consume sufficient supplemental water). The results reported here are consistent with the clinical study reports for OSS which showed few adverse events associated with its use [9]. In this study of 287,323 patients, the overall incidence for TEAEs was low (unadjusted: $<3 \%$; adjusted: $<2 \%$ ), consistent with other reports. Most prior work focused on serious adverse events and/or those with a GI basis [23-25]. For example, in a population-based, matched cohort study of over 50,000 Medicare patients who underwent outpatient colonoscopy, the overall rate of serious gastrointestinal and cardiovascular adverse events was 0.69 and $1.9 \%$, respectively [12]. However, the lower incidence of dehydration-related TEAEs associated with OSS suggests that the instructions for hydration that are provided with the product, as well as the general practice of hydration before a colonoscopy, are effective in preventing dehydration.

A possible limitation of this study is the use of a database of predominantly commercially insured beneficiaries. However, if the study data captured the true differences between study cohorts in demographic or clinical risk factors, results from the Poisson regression analysis should be applicable to a non-commercially insured population.

Another limitation is that this study was not designed to detect differences based on different mechanisms. Bowel cleansing preparations are generally categorized as being isotonic (such as PEG-ELS) or hypertonic (such as oral sodium phosphate and OSS).

As expected from prior publications, the incidences of TEAEs were low in both cohorts [12, 22-25]. The observed significant differences may not be readily detected and meaningful at the individual patient level. However, because colonoscopy is performed in a large number of patients each year, many patients are at risk and small differences equate to a meaningful effect on the population and healthcare system. For example, considering that in 2002, approximately 14 million colonoscopies were performed annually [26], each $0.1 \%$ reduction in TEAE incidence translates into 14,000 fewer events. If all bowel preparation products were associated with similar incidences of TEAEs to that of OSS, nearly 60,000 fewer TEAEs and 50,000 fewer acute cardiac conditions would be expected to occur each year.

Achieving the highest level of safety during elective colonoscopy, in otherwise generally healthy people, is a goal that demands attention. Insurance claims data provide an excellent means for measuring progress toward that goal.

The health insurance claims data only included bowel preparations requiring a prescription. However, nearly half the bowel preparations in use are over-the-counter (OTC) preparations, which are generally not FDA-approved [27-29]. A comparison of TEAEs associated with such preparations to the approved prescription products is clearly needed. Given the low incidence of TEAEs associated with colonoscopy, small changes in the incidence of an event are detectable, as shown here.

In this post-marketing study of TEAEs associated with bowel preparation and screening colonoscopy using a health insurance claims data source of 287,323 patients, the adjusted incidence of TEAEs with OSS was low $(<2 \%)$, with fewer overall events, cardiac events, and renal events than the non-OSS prescription bowel preparations. In conclusion, this study demonstrates that OSS is as safe as or possibly safer than non-OSS prescription products under actual use conditions.

Acknowledgments The authors take full responsibility for the content of and the decision to submit this manuscript.

\section{Compliance with ethical standards}

Conflict of interest Braintree Laboratories, Inc. (Braintree) sponsored this research. Covance Inc. received funding for this work. Authors include employees from Braintree, Covance Inc., and Francis A. Farraye, MD, MSc, based on their roles in study design; analysis and interpretation of data; writing and revising the manuscript; and the decision to submit the manuscript for publication. MC and RP are employees of Braintree. TK, KA, and SC are employees of Covance Inc. and received funding for this research. FAF is a scientific consultant for Braintree Laboratories, Inc. FAF was not financially compensated for collaborative efforts on publication-related activities.

Open Access This article is distributed under the terms of the Creative Commons Attribution-NonCommercial 4.0 International License (http://creativecommons.org/licenses/by-nc/4.0/), which permits any noncommercial use, distribution, and reproduction in any medium, provided you give appropriate credit to the original author(s) and the source, provide a link to the Creative Commons license, and indicate if changes were made.

\section{Appendix}

See Tables 4, 5, and 6. 
Table 4 Exhibit 1 . Treatment-emergent adverse event definitions and clinical risk factors

\begin{tabular}{lll}
\hline TEAE Definition & Clinical risk factors
\end{tabular}

Identified by diagnosis codes ${ }^{\mathrm{a}}$

Ischemic colitis $^{14} \quad$ Include:

Vascular insufficiency: 557.0, 557.1, 557.9

Exclude:

Preexisting irritable bowel disease: 564.1

Enterectomy within 14 days of vascular insufficiency: $45.6 x$, 44120, 44121

Crohn's disease within 14 days of vascular insufficiency: 555.x

Ulcerative colitis within 14 days of vascular insufficiency: 556.x

Clostridium difficile enteritis within 14 days of vascular insufficiency: 008.45

Noninfectious gastroenteritis after colonoscopy: 558.9x

5-aminosalicylate drug within 14 days of vascular insufficiency: 54569-0313, 66466-4617, 66467-4617, 66467-4617, 57866-4617, 68258-9086, 59762-0104, 59762-5000, 00179-1407, 00179-1485, 25044-0104, 25044-5000, 00904-1152, 51129-1338, 51129-1408, 51129-2976, 51129-2091, 53489-0147, 66267-0747, 66267-0747, 67651-0038, 55289-0176, 59632-0104, 59632-5700, 53002-0297, 54868-1138, 54868-1138, 54868-1139, 17856-0752, 55154-2305, 55887-0834, 66484-0752, 55289-0833, 59628-0752, 00149-0752, 00615-5515, 55154-5951, 55154-2216, 00088-2010, 54092-0189, 54092-0191, 67209-0191, 59632-0105, 00013-0105, 46603-0122, 53014-0726, 68743-0476, 67209-0476

Colonoscopy/colectomy/surgical pathology of colon biopsy: $88304,88305,88307,88309$

Aggravation of gout Gout: 274.0X, 274.1X, 274.8X, 274.9

Acute cardiac conditions or events

Abnormal electrocardiogram: 794.31

Acute myocardial infarction: 410.X0, 410.X1, 410.X2

Angina: 413.0, 413.1, 413.9

Arrhythmias: 427.0, 427.1, 427.2, 427.3X, 427.4X, 427.6X, $427.8 X, 427.9,785.0$

Cardiac arrest: 427.5

Congestive heart failure: 428.X, 428.2X, 428.3X, 428.4X

Hypotension: 458.0, 458.1, 458.21, 458.29, 458.8, 458.9

Long QT syndrome: 426.82

Shock: $785.5 \mathrm{X}$

Syncope/hypotensive syncope: 780.2

Respiratory arrest: 799.1
Heart failure: $428.0-428.4 ; 428.9$

Hyperlipidemia: 272.0-272.4

Hypotension: $458 . \mathrm{X}$

Vasculitis: 273.2; 287.0; 447.6; 447.8; 695.2

Previous abdominal surgery

Colectomy: (a) CPT: 44139; 44140; 44141; 44143; 44144; 44145 ; 44146; 44147; 44150; 44151; 44155; 44156; 44157; 44158; 44160; 44204; 44205; 44206; 44207; 44208; 44210; 44211; 44212; 44213; 44799; 45121; b) ICD-9-CM: 17.31; $17.32 ; 17.33 ; 17.34 ; 17.35 ; 17.36 ; 17.39 ; 45.71 ; 45.72 ; 45.73$; $45.74 ; 45.75 ; 45.76 ; 45.79 ; 45.81 ; 45.82 ; 45.83$

Colostomy: a) CPT: 44141; 44143; 44144; 44146; 44188; 44206; 44207; 44208; 44320; 44322; 44340; 44345; 44346; 44605; 44799; 45110; 45111; 45123; 45126; 45395; 45563; $45805 ; 45825 ; 46710 ; 46712 ; 50810 ; 51597 ; 57307 ; 57308$; 58240; 88304; 99505; (b) ICD-9-CM: 45.41; 45.43; 45.6; $46.03 ; 46.1 ; 46.10 ; 46.11 ; 46.13 ; 46.14 ; 46.23 ; 46.32 ; 46.39$; $46.42 ; 46.43 ; 46.52 ; 46.85 ; 48.5 ; 48.62 ; 68.8 ; 89.33$

Gastric bypass: (a) CPT: 43405; 43610; 43611; 43644; 43645; 43647; 43648; 43651; 43652; 43653; 43659; 43752; 43753; 43754; 43755; 43756; 43757; 43760; 43761; 43770; 43771; 43772; 43773; 43774; 43775; 43800; 43810; 43820; 43825; 43830; 43831; 43832; 43840; 43845; 43846; 43847; 43848; (b) ICD-9-CM: 43.89; 44.31; 44.38; 44.39; 44.91

Stomach stapling: (a) CPT: 43405; 43610; 43611; 43644; 43846; 43847; b) ICD-9-CM: 44.91

Arteriosclerosis: 414.0; 414.3; 440.0-440.4; 440.8; 440.9

Diabetes mellitus: 249.X; 250.X

Hyperlipidemia: 272.0-272.4

Hypertension: 401.0; 401.1; 401.9; 402.X; 404.X; 405.0; 405.1; 405.9

Thiazide diuretics use: bendroflumethiazide, chlorothiazide, hydrochlorothiazide, indapamide, metolazone, methyclothiazide, polythiazide, quinethazone, trichlormethiazide

Diabetes mellitus: 249.X; 250.X

Hyperlipidemia: $272.0-272.4$

Hypertension: 401.0; 401.1; 401.9; 402.X; 404.X; 405.0; 405.1; 405.9

Thyroid disease: $240.0 ; 240.9 ; 241.0 ; 241.1 ; 241.9$; $242.0-242.4 ; 242.8 ; 242.9 ; 243 ; 244.0-244.3 ; 244.8 ; 244.9$; $245.0-245.4 ; 245.8 ; 245.9 ; 246.0-246.3 ; 246.8 ; 246.9$

Obesity: 278.00, 278.01 
Table 4 continued

\begin{tabular}{|c|c|c|}
\hline TEAE & Definition & Clinical risk factors \\
\hline $\begin{array}{l}\text { Renal failure and } \\
\text { other serious renal } \\
\text { diseases }\end{array}$ & $\begin{array}{l}\text { Chronic kidney disease: } 585.1,585.2,585.3,585.4,585.5 \text {, } \\
585.6,585.9 \\
\text { Acute kidney injury/acute renal failure/acute phosphate } \\
\text { nephropathy : } 584.5-584.9 \\
\text { Unspecified renal failure: } 586\end{array}$ & $\begin{array}{l}\text { Diabetes mellitus: } 249 . \mathrm{X} ; 250 . \mathrm{X} \\
\text { Heart failure: } 428.0-428.4 ; 428.9 \\
\text { Hypertension: } 401.0 ; 401.1 ; 401.9 ; 402 . \mathrm{X} ; 404 . \mathrm{X} ; 405.0 ; 405.1 \text {; } \\
\quad 405.9 \\
\text { Liver disease: } 570 ; 571.0-571.6 ; 571.8 ; 571.9 ; 572.0-572.4 ; \\
\quad 572.8 ; 573.0-573.4 ; 573.8 ; 573.9 \\
\text { Peripheral artery disease: } 443.9\end{array}$ \\
\hline Seizure disorders & $\begin{array}{l}\text { Tonic-clonic seizures: } 345.10,345.11,345.3 \\
\text { Loss of consciousness: } 780.2\end{array}$ & $\begin{array}{l}\text { Stroke: 431, 432.0; 432.1; 432.9; 433.0-433.3; 433.8; 433.9; } \\
\text { 434.0; 434.1; 434.9; 435.0-435.3; 435.8; 435.9; 436, } \\
\text { 437.0-437.9; 438.0-438.9 } \\
\text { Brain cancer: } 191 . \mathrm{X}\end{array}$ \\
\hline $\begin{array}{l}\text { Serum electrolyte } \\
\text { abnormalities }\end{array}$ & $\begin{array}{l}\text { Electrolyte imbalance: } 276.9 \\
\text { Hypercalcemia: } 275.42 \\
\text { Hypocalcemia: } 275.41 \\
\text { Hypokalemia: } 276.8 \\
\text { Hypernatremia: } 276.0 \\
\text { Hyponatremia: } 276.1 \\
\text { Hypermagnesemia: } 275.2\end{array}$ & $\begin{array}{l}\text { Brain cancer: } 191 . \mathrm{X} \\
\text { Heart failure: } 428.0-428.4 ; 428.9 \\
\text { Renal disease: } 585.1,585.2,585.3,585.4,585.5,585.6,585.9 \text {, } \\
\text { 584.5-584.9, 586 } \\
\text { Thiazide diuretics use: bendroflumethiazide, chlorothiazide, } \\
\text { hydrochlorothiazide, indapamide, metolazone, } \\
\text { methyclothiazide, polythiazide, quinethazone, } \\
\text { trichlormethiazide }\end{array}$ \\
\hline
\end{tabular}

Hypomagnesemia: 275.2

Hyperphosphatemia: 275.3

Osmolality (high): 276.0

Osmolality (low): 276.1

Identified by laboratory values ${ }^{\mathrm{b}}$

$\begin{array}{lll}\text { Troponin leak } & \text { Troponin } T:>0.09 \mathrm{ng} / \mathrm{mL} & \text { None } \\ \begin{array}{l}\text { Elevated creatine } \\ \text { kinase }\end{array} & \text { Creatine kinase: }>400 \mathrm{U} /(\text { males), } 150 \mathrm{U} / \mathrm{L} \text { (females) } & \text { None }\end{array}$

Serum electrolyte abnormalities and serum uric acid
Anion gap $=[\mathrm{Na}+\mathrm{K}]-\left([\mathrm{Cl}-]+\left[\mathrm{HCO}_{3}-\right]\right)($ high $)$ : $>16 \mathrm{mmol} / \mathrm{L}$

Bicarbonate (low): $<23 \mathrm{mmol} / \mathrm{L}$

Bilirubin, total (high): $>1 \mathrm{mg} / \mathrm{dL}$

BUN (high): $>25 \mathrm{mg} / \mathrm{dL}$

Calcium (low): $<8.5 \mathrm{mg} / \mathrm{dL}$

Calcium (high): $>10.5 \mathrm{mg} / \mathrm{dL}$

Chloride (high): $>108 \mathrm{mmol} / \mathrm{L}$

Creatinine (high): $>1.5 \mathrm{mg} / \mathrm{dL}$

Magnesium (low): $<1.4 \mathrm{mEq} / \mathrm{L}$

Magnesium (high): $>2.0 \mathrm{mEq} / \mathrm{L}$

Osmolality (low): $<280 \mathrm{mOsm} / \mathrm{kg}$

Osmolality (high): $>296 \mathrm{mOsm} / \mathrm{kg}$

Phosphorus (high): $>4.5 \mathrm{mg} / \mathrm{dL}$

Potassium (low): $<3.4 \mathrm{mmol} / \mathrm{L}$

Sodium (low): $<135 \mathrm{mmol} / \mathrm{L}$

Sodium (high): $>145 \mathrm{mmol} / \mathrm{L}$

Uric acid elevations: $>8.5 \mathrm{mg} / \mathrm{dL}$ (males), $6.6 \mathrm{mg} / \mathrm{dL}$ (females)
None

Electrolyte abnormalities:

Brain cancer: 191.X

Heart failure: $428.0-428.4 ; 428.9$

Renal disease: 585.1, 585.2, 585.3, 585.4, 585.5, 585.6, 585.9, 584.5-584.9, 586

Thiazide diuretics use: bendroflumethiazide, chlorothiazide, hydrochlorothiazide, indapamide, metolazone, methyclothiazide, polythiazide, quinethazone, trichlormethiazide

Uric acid:

Arteriosclerosis: 414.0; 414.3; 440.0-440.4; 440.8; 440.9

Diabetes mellitus: 249.X; 250.X

Gout: 274.0X, 274.1X, 274.8X, 274.9

Hyperlipidemia: 272.0-272.4

Hypertension: 401.0; 401.1; 401.9; 402.X; 404.X; 405.0; 405.1; 405.9

Obesity: 278.00, 278.01

Thiazide diuretics use: bendroflumethiazide, chlorothiazide, hydrochlorothiazide, indapamide, metolazone, methyclothiazide, polythiazide, quinethazone, trichlormethiazide

Renal disease: 585.1, 585.2, 585.3, 585.4, 585.5, 585.6, 585.9, $584.5-584.9,586$

\footnotetext{
${ }^{a}$ For ischemic colitis, CPT and ICD-9-CM procedure codes for enterectomy and colonoscopy/colectomy/surgical pathology of colon biopsy also were used in addition to ICD-9-CM diagnosis codes. National Drug Code was used to identify 5-aminosalicylate drugs

b Out-of-range values from Massachusetts General Hospital (MGH) Department of Radiology, MGH Laboratory Handbook: Reference Intervals-MGH Clinical Laboratories. Report generated March 23, 2011. Online Lab Handbook: http://mghlabtest.partners.org
} 
Table 5 Exhibit 2. Unadjusted incidence of treatment-emergent adverse events comprising the overall categories

\begin{tabular}{|c|c|c|c|c|c|c|}
\hline \multirow[t]{2}{*}{ Treatment-emergent adverse event (TEAE) } & \multicolumn{3}{|c|}{$\operatorname{SUPREP}(N=31,885)$} & \multicolumn{3}{|c|}{ Control $(N=255,438)$} \\
\hline & $n$ & Incidence $(\%)$ & $95 \% \mathrm{CI}$ & $n$ & Incidence $(\%)$ & $95 \% \mathrm{CI}$ \\
\hline \multicolumn{7}{|l|}{ Identified by diagnosis codes } \\
\hline \multicolumn{7}{|l|}{ Acute cardiac conditions or events } \\
\hline Abnormal electrocardiogram & 141 & 0.44 & $0.38,0.52$ & 1465 & 0.57 & $0.54,0.60$ \\
\hline Acute myocardial infarction & 21 & 0.07 & $0.04,0.10$ & 173 & 0.07 & $0.06,0.08$ \\
\hline Angina & 46 & 0.14 & $0.11,0.19$ & 395 & 0.15 & $0.14,0.17$ \\
\hline Arrhythmias & 230 & 0.72 & $0.63,0.82$ & 2250 & 0.88 & $0.85,0.92$ \\
\hline Cardiac arrest & 1 & 0.00 & $0.00,0.02$ & 27 & 0.01 & $0.01,0.02$ \\
\hline Congestive heart failure & 28 & 0.09 & $0.06,0.13$ & 339 & 0.13 & $0.12,0.15$ \\
\hline Hypotension & 30 & 0.09 & $0.07,0.13$ & 309 & 0.12 & $0.11,0.14$ \\
\hline Long QT syndrome & 0 & 0.00 & $0.00,0.01$ & 9 & 0.00 & $0.00,0.01$ \\
\hline Shock & 5 & 0.02 & $0.01,0.04$ & 50 & 0.02 & $0.01,0.03$ \\
\hline Syncope/hypotensive syncope & 69 & 0.22 & $0.17,0.27$ & 665 & 0.26 & $0.24,0.28$ \\
\hline Respiratory arrest & 0 & 0.00 & $0.00,0.01$ & 9 & 0.00 & $0.00,0.01$ \\
\hline \multicolumn{7}{|l|}{ Renal failure and other serious renal diseases } \\
\hline Chronic kidney disease & 42 & 0.13 & $0.10,0.18$ & 532 & 0.21 & $0.19,0.23$ \\
\hline Acute kidney failure & 26 & 0.08 & $0.06,0.12$ & 317 & 0.12 & $0.11,0.14$ \\
\hline Unspecified renal failure & 12 & 0.04 & $0.02,0.07$ & 92 & 0.04 & $0.03,0.04$ \\
\hline \multicolumn{7}{|l|}{ Seizure disorders } \\
\hline Tonic-clonic seizures & 3 & 0.01 & $0.00,0.03$ & 41 & 0.02 & $0.01,0.02$ \\
\hline Loss of consciousness & 82 & 0.26 & $0.21,0.32$ & 821 & 0.32 & $0.30,0.34$ \\
\hline \multicolumn{7}{|l|}{ Serum electrolyte abnormalities } \\
\hline Electrolyte imbalance & 17 & 0.05 & $0.03,0.09$ & 108 & 0.04 & $0.04,0.05$ \\
\hline Hypercalcemia & 17 & 0.05 & $0.03,0.09$ & 191 & 0.07 & $0.06,0.09$ \\
\hline Hypocalcemia & 10 & 0.03 & $0.02,0.06$ & 61 & 0.02 & $0.02,0.03$ \\
\hline Hypokalemia & 55 & 0.17 & $0.13,0.22$ & 573 & 0.22 & $0.21,0.24$ \\
\hline Hypermagnesemia or hypomagnesemia & 8 & 0.03 & $0.01,0.05$ & 112 & 0.04 & $0.04,0.05$ \\
\hline Hyperphosphatemia & 0 & 0.00 & $0.00,0.01$ & 34 & 0.01 & $0.01,0.02$ \\
\hline Hyperosmolality and/or hypernatremia & 4 & 0.01 & $0.00,0.03$ & 32 & 0.01 & $0.01,0.02$ \\
\hline Hyposmolality and/or hyponatremia & 27 & 0.08 & $0.06,0.12$ & 275 & 0.11 & $0.10,0.12$ \\
\hline Identified by laboratory values & 653 & & & 4,432 & & \\
\hline \multicolumn{7}{|c|}{ Serum electrolyte abnormalities and serum uric acid } \\
\hline Anion gap (high) & 0 & 0.00 & $0.00,0.56$ & 4 & 0.03 & $0.01,0.07$ \\
\hline Bicarbonate (low) & 0 & 0.00 & $0.00,0.56$ & 3 & 0.02 & $0.01,0.06$ \\
\hline Bilirubin, total (high) & 0 & 0.00 & $0.00,0.56$ & 40 & 0.28 & $0.20,0.38$ \\
\hline BUN (high) & 3 & 0.46 & $0.15,1.42$ & 54 & 0.37 & $0.29,0.49$ \\
\hline Calcium (low) & 1 & 0.15 & $0.02,1.08$ & 3 & 0.02 & $0.01,0.06$ \\
\hline Calcium (high) & 0 & 0.00 & $0.00,0.56$ & 16 & 0.11 & $0.07,0.18$ \\
\hline Chloride (high) & 0 & 0.00 & $0.00,0.56$ & 26 & 0.18 & $0.12,0.26$ \\
\hline Creatinine (high) & 0 & 0.00 & $0.00,0.56$ & 13 & 0.09 & $0.05,0.16$ \\
\hline Magnesium (low) & 1 & 0.15 & $0.02,1.08$ & 19 & 0.13 & $0.08,0.21$ \\
\hline Magnesium (high) & 0 & 0.00 & $0.00,0.56$ & 6 & 0.04 & $0.02,0.09$ \\
\hline Osmolality (low) & 0 & 0.00 & $0.00,0.56$ & 2 & 0.01 & $0.00,0.06$ \\
\hline Osmolality (high) & 0 & 0.00 & $0.00,0.56$ & 0 & 0.00 & $0.00,0.03$ \\
\hline Phosphorus (high) & 0 & 0.00 & $0.00,0.56$ & 2 & 0.01 & $0.00,0.06$ \\
\hline Potassium (low) & 1 & 0.15 & $0.02,1.08$ & 22 & 0.15 & $0.10,0.23$ \\
\hline Sodium (low) & 0 & 0.00 & $0.00,0.56$ & 19 & 0.13 & $0.08,0.21$ \\
\hline Sodium (high) & 0 & 0.00 & $0.00,0.56$ & 9 & 0.06 & $0.03,0.12$ \\
\hline
\end{tabular}


Table 5 continued

\begin{tabular}{|c|c|c|c|c|c|c|}
\hline \multirow[t]{2}{*}{ Treatment-emergent adverse event (TEAE) } & \multicolumn{3}{|c|}{ SUPREP $(N=31,885)$} & \multicolumn{3}{|c|}{ Control $(N=255,438)$} \\
\hline & $n$ & Incidence $(\%)$ & $95 \% \mathrm{CI}$ & $n$ & Incidence $(\%)$ & $95 \%$ CI \\
\hline Uric acid elevations & 0 & 0.00 & $0.00,0.56$ & 7 & 0.05 & $0.02,0.10$ \\
\hline
\end{tabular}

The unadjusted cumulative incidence of each TEAE was calculated using the Kaplan-Meier method. When there were no events observed, the Clopper-Pearson method was used to calculate exact CIs

CI confidence interval

Table 6 Exhibit 3. Adjusted incidence of treatment-emergent adverse events comprising the overall categories

\begin{tabular}{|c|c|c|c|c|c|c|}
\hline \multirow[t]{2}{*}{ Treatment-emergent adverse event } & \multicolumn{3}{|c|}{ OSS $(N=31,885)$} & \multicolumn{3}{|c|}{ Non-OSS $(N=255,438)$} \\
\hline & $n$ & Incidence $(\%)$ & $95 \% \mathrm{CI}$ & $n$ & Incidence $(\%)$ & $95 \% \mathrm{CI}$ \\
\hline \multicolumn{7}{|l|}{ Identified by diagnosis codes } \\
\hline \multicolumn{7}{|l|}{ Acute cardiac conditions or events } \\
\hline Abnormal electrocardiogram & 141 & 0.36 & $0.30,0.42$ & 1465 & 0.46 & $0.42,0.49$ \\
\hline Acute myocardial infarction & 21 & 0.05 & $0.03,0.08$ & 173 & 0.05 & $0.04,0.06$ \\
\hline Angina & 46 & 0.08 & $0.06,0.12$ & 395 & 0.09 & $0.07,0.10$ \\
\hline Arrhythmias & 230 & 0.63 & $0.55,0.72$ & 2250 & 0.76 & $0.72,0.80$ \\
\hline Cardiac arrest & 1 & 0.00 & $0.00,0.01$ & 27 & 0.01 & $0.00,0.01$ \\
\hline Congestive heart failure & 28 & 0.05 & $0.03,0.07$ & 339 & 0.07 & $0.05,0.08$ \\
\hline Hypotension & 30 & 0.07 & $0.05,0.10$ & 309 & 0.09 & $0.07,0.10$ \\
\hline Long QT syndrome & 0 & 0.00 & $0.00,0.01$ & 9 & 0.00 & $0.00,0.01$ \\
\hline Shock & 5 & 0.02 & $0.01,0.04$ & 50 & 0.02 & $0.01,0.03$ \\
\hline Syncope/hypotensive syncope & 69 & 0.17 & $0.14,0.22$ & 665 & 0.21 & $0.18,0.23$ \\
\hline Respiratory arrest & 0 & 0.00 & $0.00,0.01$ & 9 & 0.00 & $0.00,0.01$ \\
\hline \multicolumn{7}{|l|}{ Renal failure and other serious renal diseases } \\
\hline Chronic kidney disease & 42 & 0.07 & $0.05,0.09$ & 532 & 0.10 & $0.08,0.11$ \\
\hline Acute kidney failure & 26 & 0.04 & $0.02,0.06$ & 317 & 0.05 & $0.04,0.06$ \\
\hline Unspecified renal failure & 12 & 0.02 & $0.01,0.03$ & 92 & 0.02 & $0.01,0.02$ \\
\hline \multicolumn{7}{|l|}{ Seizure disorders } \\
\hline Tonic-clonic seizures & 3 & 0.01 & $0.00,0.03$ & 41 & 0.02 & $0.01,0.02$ \\
\hline Loss of consciousness & 82 & 0.25 & $0.20,0.31$ & 821 & 0.31 & $0.29,0.33$ \\
\hline \multicolumn{7}{|l|}{ Serum electrolyte abnormalities } \\
\hline Electrolyte imbalance & 17 & 0.05 & $0.03,0.08$ & 108 & 0.04 & $0.03,0.05$ \\
\hline Hypercalcemia & 17 & 0.04 & $0.03,0.07$ & 191 & 0.06 & $0.05,0.07$ \\
\hline Hypocalcemia & 10 & 0.03 & $0.01,0.05$ & 61 & 0.02 & $0.01,0.02$ \\
\hline Hypokalemia & 55 & 0.14 & $0.10,0.18$ & 573 & 0.17 & $0.15,0.19$ \\
\hline Hypermagnesemia or hypomagnesemia & 8 & 0.02 & $0.01,0.05$ & 112 & 0.04 & $0.03,0.05$ \\
\hline Hyperphosphatemia & 0 & 0.00 & $0.00,0.01$ & 34 & 0.01 & $0.00,0.01$ \\
\hline Hyperosmolality and/or hypernatremia & 4 & 0.01 & $0.00,0.03$ & 32 & 0.01 & $0.01,0.02$ \\
\hline Hyposmolality and/or hyponatremia & 27 & 0.08 & $0.05,0.11$ & 275 & 0.10 & $0.08,0.11$ \\
\hline Identified by laboratory values & 653 & & & 4,432 & & \\
\hline \multicolumn{7}{|c|}{ Serum electrolyte abnormalities and serum uric acid } \\
\hline Anion gap (high) & 0 & 0.00 & $0.00,0.56$ & 4 & 0.03 & $0.01,0.07$ \\
\hline Bicarbonate (low) & 0 & 0.00 & $0.00,0.56$ & 3 & 0.01 & $0.00,0.06$ \\
\hline Bilirubin, total (high) & 0 & 0.00 & $0.00,0.56$ & 40 & 0.24 & $0.17,0.35$ \\
\hline BUN (high) & 3 & 0.38 & $0.12,1.20$ & 54 & 0.30 & $0.22,0.41$ \\
\hline Calcium (low) & 1 & 0.09 & $0.01,0.77$ & 3 & 0.01 & $0.00,0.05$ \\
\hline
\end{tabular}


Table 6 continued

\begin{tabular}{|c|c|c|c|c|c|c|}
\hline \multirow[t]{2}{*}{ Treatment-emergent adverse event } & \multicolumn{3}{|c|}{ OSS $(N=31,885)$} & \multicolumn{3}{|c|}{ Non-OSS $(N=255,438)$} \\
\hline & $n$ & Incidence $(\%)$ & $95 \% \mathrm{CI}$ & $n$ & Incidence $(\%)$ & $95 \% \mathrm{CI}$ \\
\hline Calcium (high) & 0 & 0.00 & $0.00,0.56$ & 16 & 0.08 & $0.04,0.15$ \\
\hline Chloride (high) & 0 & 0.00 & $0.00,0.56$ & 26 & 0.18 & $0.12,0.26$ \\
\hline Creatinine (high) & 0 & 0.00 & $0.00,0.56$ & 13 & 0.06 & $0.03,0.11$ \\
\hline Magnesium (low) & 1 & 0.15 & $0.02,1.05$ & 19 & 0.12 & $0.08,0.19$ \\
\hline Magnesium (high) & 0 & 0.00 & $0.00,0.56$ & 6 & 0.04 & $0.02,0.09$ \\
\hline Osmolality (low) & 0 & 0.00 & $0.00,0.56$ & 2 & 0.01 & $0.00,0.05$ \\
\hline Osmolality (high) & 0 & 0.00 & $0.00,0.56$ & 0 & 0.00 & $0.00,0.03$ \\
\hline Phosphorus (high) & 0 & 0.00 & $0.00,0.56$ & 2 & 0.01 & $0.00,0.06$ \\
\hline Potassium (low) & 1 & 0.12 & $0.02,0.88$ & 22 & 0.11 & $0.07,0.19$ \\
\hline Sodium (low) & 0 & 0.00 & $0.00,0.56$ & 19 & 0.13 & $0.08,0.21$ \\
\hline Sodium (high) & 0 & 0.00 & $0.00,0.56$ & 9 & 0.06 & $0.03,0.12$ \\
\hline Uric acid elevations & 0 & 0.00 & $0.00,0.56$ & 7 & 0.02 & $0.01,0.06$ \\
\hline
\end{tabular}

Adjusted incidence was calculated using Poisson regression, controlling for patient factors including demographic characteristics and clinical risk factors. Differences between the two cohorts were tested using the Wald Chi-square. A patient factor was removed from the Poisson model if the parameter estimate was not significant (i.e., $p$ value greater than 0.10 ). When there were no events observed, the Clopper-Pearson method was used to calculate exact CIs. The Fisher's exact test was used to calculate the $p$ value for troponin leak

CI confidence interval

\section{References}

1. Rex DK, Johnson DA, Anderson JC, et al. American College of Gastroenterology guidelines for colorectal cancer screening 2008 [corrected]. Am J Gastroenterol. 2009;104:739-750.

2. Fisher DA, Maple JT, Ben-Menachem T, et al. ASGE standards of practice committee, complications of colonoscopy. Gastrointest Endosc. 2011;74:745-752.

3. Johnson DA, Barkun AN, Cohen LB, et al. Optimizing adequacy of bowel cleansing for colonoscopy: recommendations from the US Multi-Society Task Force on Colorectal Cancer. US MultiSociety Task Force on Colorectal Cancer. Am J Gastroenterol. 2014;109:1528-1545.

4. Davis GL, Santa Ana CA, Morawski SG, et al. Development of a lavage solution associated with minimal water and electrolyte absorption or secretion. Gastroenterology. 1980;78:991-995.

5. Fordtran JS, Santa Ana C, Cleveland MVB. A low sodium solution for gastrointestinal lavage. Gastroenterology. 1990;98:11-16.

6. Vanner SJ, MacDonald PH, Paterson WC, et al. A randomized prospective trial comparing oral sodium phosphate with standard polyethylene glycol-based lavage solution (Golytely) in preparation of patients for colonoscopy. Am J Gastro. 1990;85:422-427.

7. Markowitz GS, Stokes MB, Radhakrishnan J, et al. Acute phosphate nephropathy following oral sodium phosphate bowel purgative: an underrecognized cause of chronic renal failure. $J$ Am Soc Nephrol. 2005;16:3389-3396.

8. United States Food and Drug Association FDA Alert on "Information for Healthcare Professionals: Oral Sodium Phosphate (OSP) Products for Bowel Cleansing (marketed as Visicol and OsmoPrep, and oral sodium phosphate products available without a prescription)" 2008 [Accessed May 6, 2016]. Available from: http://www.fda.gov/Drugs/DrugSafety/PostmarketDrugSafetyIn formationforPatientsandProviders/ucm126084.htm.

9. DiPalma JA, Rodriguez R, McGowan J, et al. A randomized clinical study evaluating the safety and efficacy of a new, reduced-volume, oral sulfate colon-cleansing preparation for colonoscopy. Am J Gastro. 2009;104:2275-2284.
10. Patel V, Nicar M, Emmett M, et al. Intestinal and renal effects of low-volume phosphate and sulfate cathartic solutions designed for cleansing the colon: pathophysiological studies in five normal subjects. Am J Gastro. 2009;104:953-965.

11. Rex DK, Di Palma JA, Rodriguez R, et al. A randomized clinical study comparing reduced-volume oral sulfate solution with standard 4-liter sulfate-free electrolyte lavage solution as preparation for colonoscopy. Gastrointest Endosc. 2010;72:328-336.

12. Warren JL, Klabunde CN, Marlotto AB, et al. Adverse events after outpatient colonoscopy in the medicare population. Ann Intern Med. 2009;150:849-857.

13. Massachusetts General Hospital (MGH) Department of Radiology [Internet]. MGH Laboratory Handbook: Reference Intervals-MGH Clinical Laboratories [cited 2011 March 23]. Available from: http://mghlabtest.partners.org.

14. Sands BE, Duh M-S, Cali C, et al. Algorithms to identify colonic ischemia, complications of constipation and irritable bowel syndrome in medical claims data: development and validation. Pharmacoepidemiol Drug Safety. 2006;15:47-56.

15. Mayo Foundation for Medical Education and Research[Internet]. Risk factors sections listed for each adverse event [cited 2011 September 30]. Available from: http://www.mayoclinic.com/ health-information.

16. Upton G, Cook I. A dictionary of statistics. 3rd ed. New York: Oxford University Press; 2014.

17. Yoav B, Yosef H. Controlling the false discovery rate: a practical and powerful approach to multiple testing. J R Stat Soc B. 1995;57:289-300.

18. Kelsey JL, Whittemore AS, Evans AS, Thompson WD. Methods in observational epidemiology. 2nd ed. New York: Oxford University Press; 1996.

19. Wilchesky M, Tamblyn RM, Huang A. Validation of diagnostic codes within medical services claims. $J$ Clin Epidemiol. 2004;57:131-141.

20. Schneeweiss S, Avorn A. A review of uses of health care utilization databases for epidemiologic research on therapeutics. $J$ Clin Epidemiol. 2005;58:323-337. 
21. Pelham RW, Alcorn H Jr, Cleveland MVB. A pharmacokinetics evaluation of a new, low-volume, oral sulfate colon cleansing preparation in patients with renal or hepatic impairment and healthy volunteers. J Clin Pharmacol. 2010;50:350-354.

22. Pelham R, Jongenelen I, Cleveland MVB. The safety and biochemistry of an experimental low volume, oral sulfate colonoscopy preparation compared to a phosphate-based preparation in humans. Gastroenterology. 2009;136:T2028.2009.

23. Rutter CM, Johnson E, Miglioretti DL, et al. Adverse events after screening and follow-up colonoscopy. Cancer Causes Control. 2012;23:289-296.

24. Ho JM, Gruneir A, Fischer HD, et al. Serious events in older Ontario residents receiving bowel preparations for outpatient colonoscopy with various comorbidity profiles: a descriptive, population-based study. Can J Gastroenterol. 2012;26:436-440.

25. Levin TR, Zhao W, Conell C, et al. Complications of colonoscopy in an integrated health care delivery system. Ann Intern Med. 2006;145:880-886.
26. Seeff LC, Richards TB, Shapiro JA, et al. How many endoscopies are performed for colorectal cancer screening? Results from CDC's survey of endoscopic capacity. Gastroenterology. 2004;127:1670-1677.

27. Shieh FK, Gunaratnam N, Mohamud SO, et al. MiraLAX-Gatorade bowel prep versus GoLytely before screening colonoscopy: an endoscopic database study in a community hospital. $J$ Clin Gastroenterol. 2012;46:e96-e100.

28. Samarasena JB, Muthusamy VR, Jamal MM. Split-dosed MiraLAX/Gatorade is an effective, safe, and tolerable option for bowel preparation in low-risk patients: a randomized controlled study. Am J Gastroenterol. 2012;107:1036-1042.

29. Enestvedt BK, Brian Fennerty M, Zaman A, et al. MiraLAX vs. Golytely: is there a significant difference in the adenoma detection rate? Aliment Pharmacol Ther. 2011;34:775-782. 